\title{
Generating Mutually Inductive Theorems from Concise Descriptions
}

\author{
Sol Swords \\ Centaur Technology, Inc. \\ sswords@centtech.com
}

\begin{abstract}
We describe defret-mutual-generate, a utility for proving ACL2 theorems about large mutually recursive cliques of functions. This builds on previous tools such as defret-mutual and make-flag, which automate parts of the process but still require a theorem body to be written out for each function in the clique. For large cliques, this tends to mean that certain common hypotheses and conclusions are repeated many times, making proofs difficult to read, write, and maintain. This utility automates several of the most common patterns that occur in these forms, such as including hypotheses based on formal names or types. Its input language is rich enough to support forms that have some common parts and some unique parts per function. One application of defret-mutual-generate has been to support proofs about the FGL rewriter, which consists of a mutually recursive clique of 49 functions. The use of this utility reduced the size of the forms that express theorems about this clique by an order of magnitude. It also greatly has reduced the need to edit theorem forms when changing definitions in the clique, even when adding or removing functions.
\end{abstract}

\section{Introduction}

Mutual recursion is used fairly frequently in ACL2, but it is still relatively rare to prove significant theorems about mutually recursive functions. Most theorems in the ACL2 community books that mention mutually recursive functions are generated by utilities such as fty: :deftypes, fty: :deffixequivmutual, or the :returns feature of std: :defines [1]. We posit that the reason for this is not that mutually recursive algorithms are uninteresting, but that perhaps few users know of the existing tools that support proofs about them. Another impediment is that it is usually necessary to write several variations of the desired theorem, one for each function in the clique, in order to prove a theorem by mutual induction.

In this paper we first describe existing processes for proving inductive theorems about mutual recursions, including the utilities make-flag and defret-mutual. We then describe a new utility, defretmutual-generate, that builds on these and automates the generation of such theorems, using schemas that address many common usage patterns. This utility was developed alongside the FGL rewriter, the core definitions of which are in a clique of 49 mutually-recursive functions. We calculate that without the use of this utility, the forms expressing the core invariants and correctness theorems about the FGL rewriter would have been an order of magnitude bigger. Furthermore, the use of this utility saves the need to edit all of these theorem forms every time a function in the clique is added, removed, or its input/output signature changed-usually most of the theorem forms can be left unmodified.

Many of the utilities described here are more thoroughly documented in the combined ACL2 and community books manual [1]. We'll refer simply to "the manual" as a shorthand when we reference the respective documentation topics for such utilities.

G. Passmore, R. Gamboa (Eds.): ACL2 Workshop 2020

EPTCS 327, 2020, pp. 95-107 doi 10.4204/EPTCS.327.10 (c) S. Swords

This work is licensed under the Creative Commons Attribution License. 
Listing 1: Definitions of subst-term, ev-term, and ev-alist

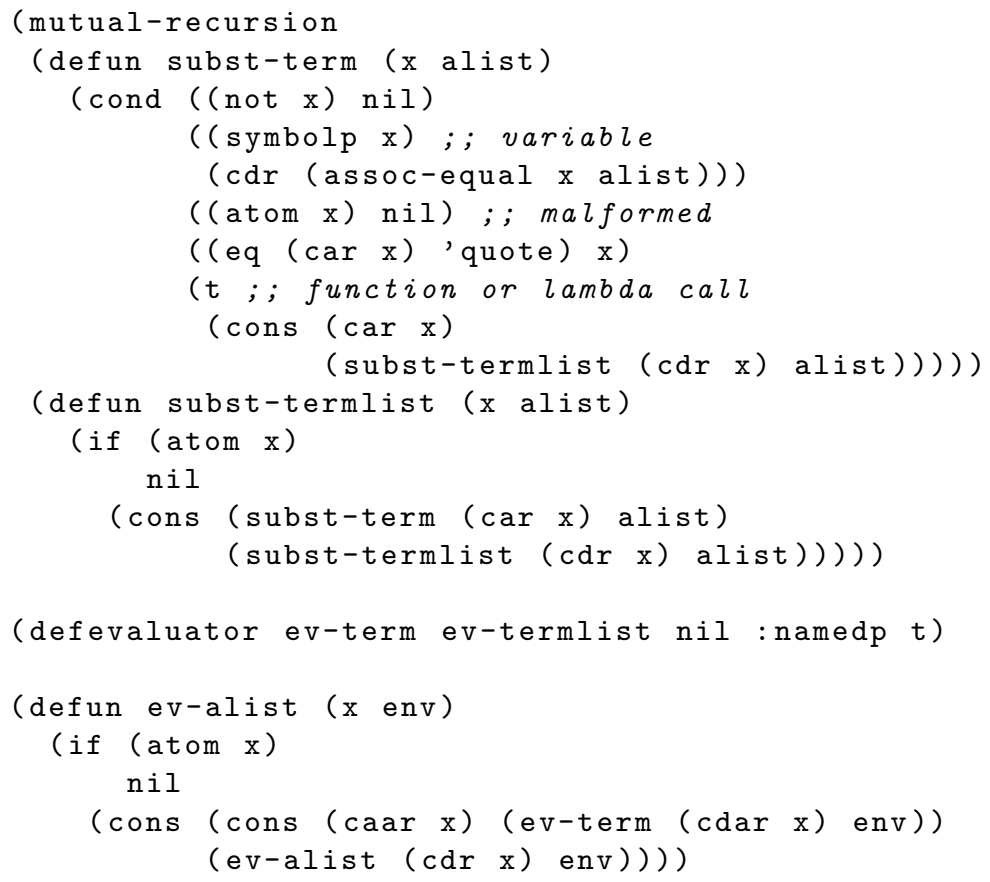

Listing 2: Evaluation of subst-term theorem

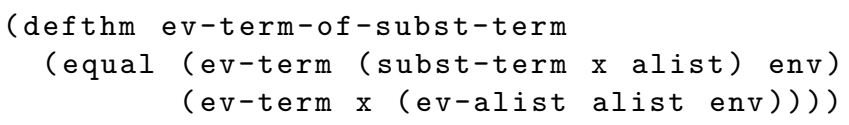

\section{Mutually Inductive Proofs}

We first show a simple mutually inductive proof about a mutually recursive clique of functions, then describe how to scale this proof strategy to more complicated functions. For our example, we'll prove a theorem about a term substitution algorithm, subst-term, defined in Listing 1

The theorem we will prove states its semantics with respect to ev-term, a standard term evaluator created with defevaluator [3]. The theorem we want is shown in Listing 2.

The problem we'll encounter if we try to prove ev-term-of-subst-term is that we need a lemma, ev-termlist-of-subst-termlist (Listing 3). But we can't prove that lemma by itself, because we need the original ev-term-of-subst-term - that is, we need to prove the two theorems via mutual induction. The simplest way to prove these two theorems is to prove their conjunction, ev-term/listof-subst-term/list (Listing 4), by an induction scheme that recurs on the car and cdr of $\mathrm{x}$.

Listing 3: Evaluation of subst-termlist theorem

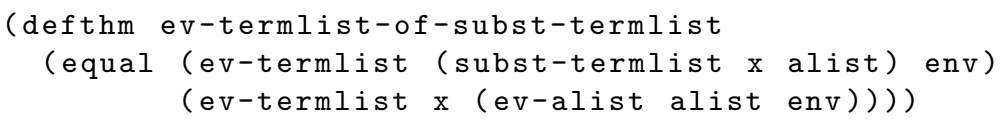




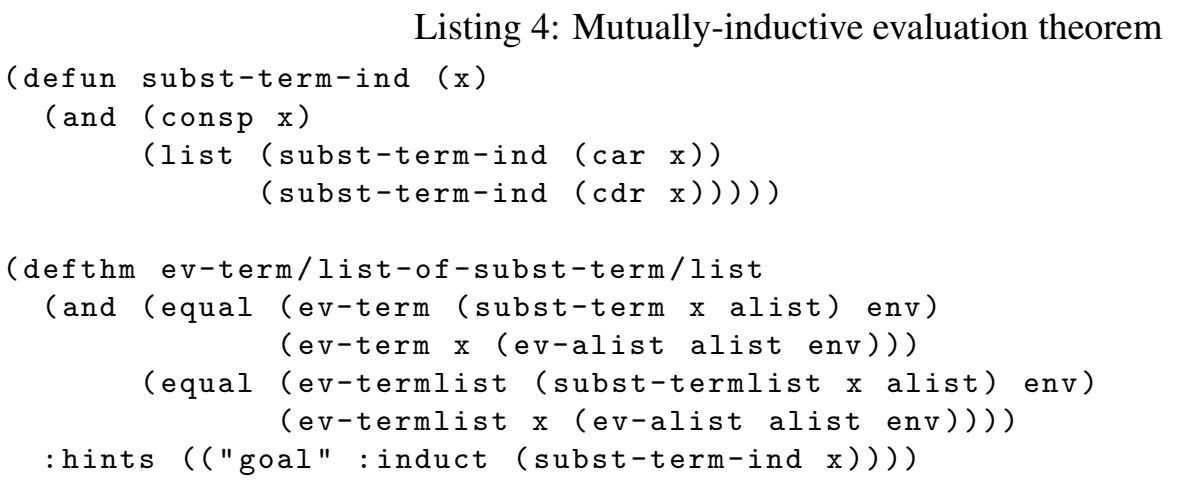

In this approach to the problem, we prove the conjunction of the mutually inductive theorems using a custom induction scheme, which typically must match the recursive calls of all the functions of the clique. Here subst-term-ind suffices because it recurs on ( $\operatorname{cdr} \mathrm{x}$ ) when $\mathrm{x}$ is a function or lambda call term, as in subst-term, and it recurs on both ( $\operatorname{car} \mathrm{x}$ ) and ( $\operatorname{cdr} \mathrm{x}$ ) when $\mathrm{x}$ is a cons, as does subst-termlist.

There are two problems with applying this approach to larger problems. First, it isn't always easy to hand-craft an induction scheme that contains a superset of all the recursive calls of a clique. Second, these sorts of induction schemes will produce too many induction hypotheses. In this example, we still have a fast proof despite generating several useless induction hypotheses. But for larger cliques, the number of induction hypotheses will usually grow as the number of functions in the clique times the number of different recursive calls, which can quickly overwhelm the prover with useless hypotheses.

These two problems can be addressed by instead doing the induction using a flag function version of the mutual recursion. Any mutually-recursive clique of functions can be transformed into a single function whose formals are the union of the formals of the clique functions along with an extra formal called the flag, which tells which function of the clique the flag function should emulate. This technique dates back at least to 1984, when Boyer and Moore [2] noted:

...it is well known that mutual recursion can be eliminated by the trick of defining a single function that has an extra "flag" argument...

The flag function can be proved equal to the functions of the original mutual recursion, dispatched by the flag. A flag function for subst-term and its equivalence theorem is shown in Listing 5 .

The flag function can then be used as an induction scheme to prove mutually-inductive theorems about the original functions, using the flag variable to distinguish between the cases. That is, instead of proving the conjunction of all the mutually inductive theorems, we prove that each of them is true when the flag is the corresponding value, as shown in Listing 6. This form of the theorem usually doesn't produce good rewrite or other rule classes because of the presence of the extra flag variable. But instantiating this theorem with the various values of the flag variable is an easy way to prove the original mutually-inductive theorems.

There are several advantages of this scheme over the previous approach of proving the conjunction using a custom induction scheme. It is easy to automate because the transformation of the clique to a flag function is straightforward. The induction scheme is specific to each function of the clique, so that after some simple case splitting, only the particular induction hypotheses needed for a given case are left. Because the flag function emulates the original clique, its induction scheme even works when there are reflexive recursive calls, that is, recursive calls on the results of other recursive calls. 
Listing 5: Flag function for subst-term

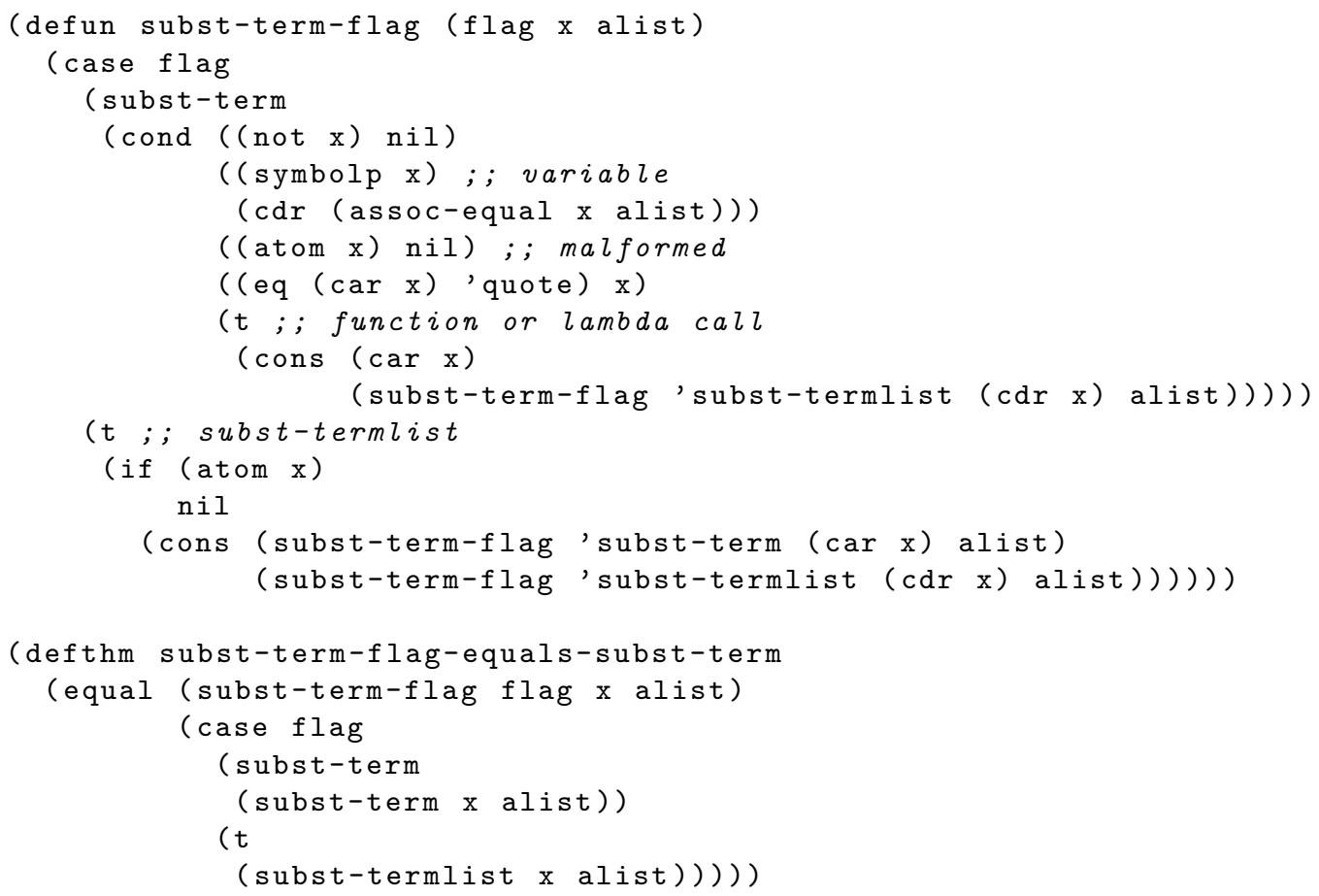

Listing 6: Flag-style proof of subst-term evaluation

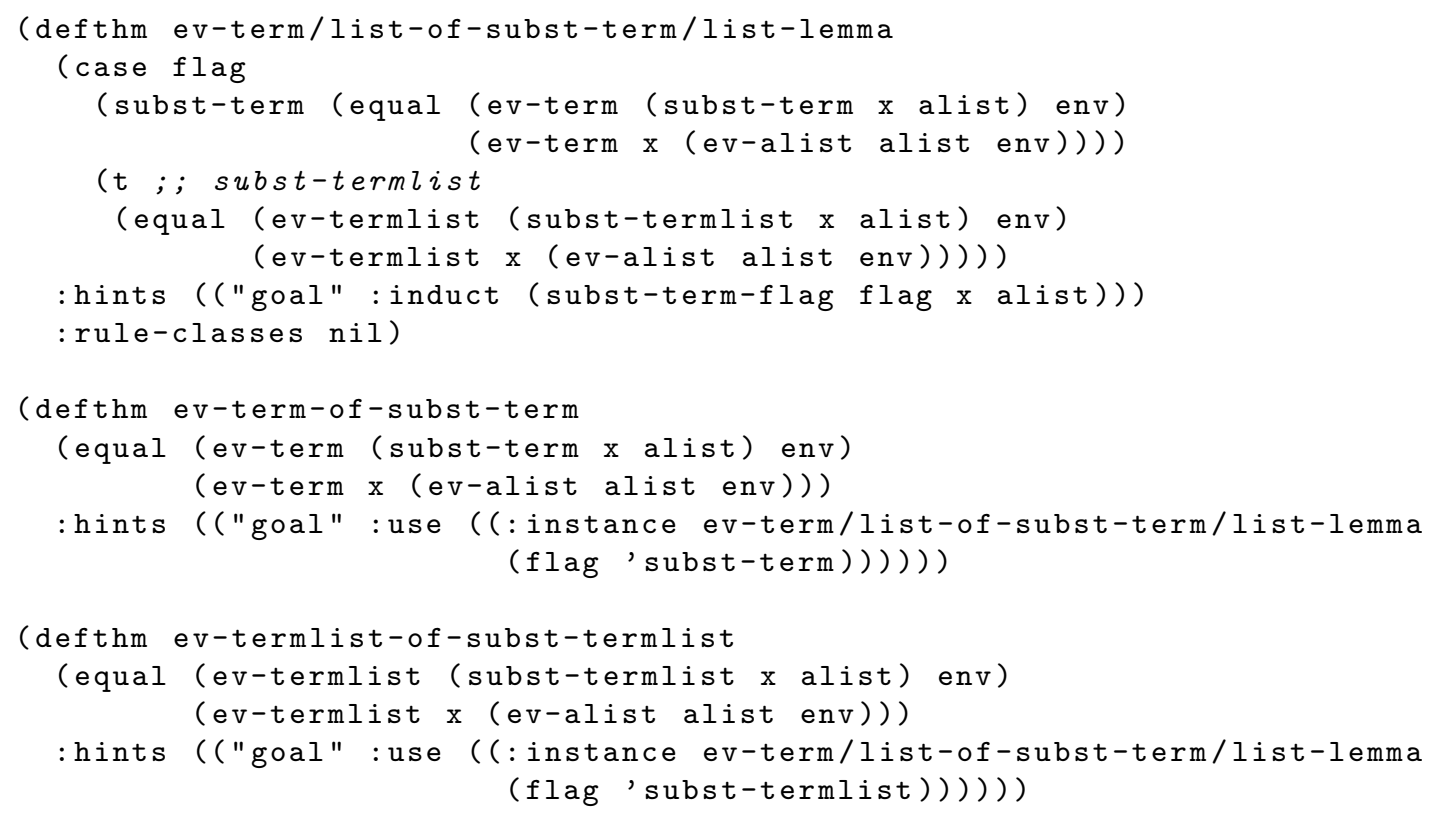




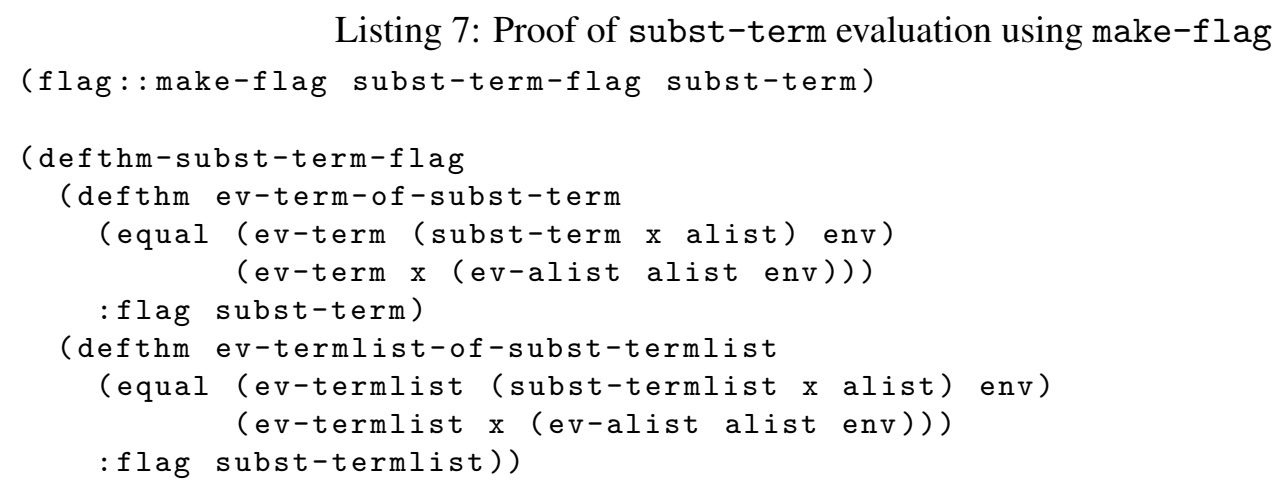

\section{Flag function method using make- $f$ lag}

The macro make-flag automates the flag function method shown in the previous section. A reference for the full feature set of make-flag is in the manual [1] and beyond the scope of this paper, but we briefly describe what it does by example.

The events of Listing 7 show how to prove the two mutually-inductive theorems of the previous section. The make-flag event admits a flag function and equivalence theorem, similar to the handcoded events of Listing 5. It also defines a new macro named defthm-subst-term-flag that uses the flag function to prove a mutually-inductive set of theorems about the original clique. We'll call this sort of macro a flag defthm macro. For each theorem, the user must specify which function of the clique (and therefore which value of the flag) it corresponds to. It generates an encapsulate event that contains essentially the events of Listing 6, with the original lemma local to the encapsulate but the other two theorems exported.

\subsection{Using defun-sk Instead of Specialized Induction Schemes}

In some cases a proof seems to require an induction scheme that isn't exactly the one generated by the main (mutually) recursive function involved. For example, in Listing 8 we show the mutually-recursive definitions of remove-return-last-term and remove-return-last-termlist and a pair of theorems about the clique (where $r l-e v$ and $r l-e v-l i s t$ are a term/list evaluator pair). Intuitively we might expect to prove these using the induction scheme of a flag function generated from the clique. However, this induction doesn't suffice to prove these theorems directly, because the induction hypothesis we need for the lambda case has a substitution for env as well as for $\mathrm{x}$.

One way to solve this problem is to write a custom induction scheme that produces the same substitutions for $\mathrm{x}$ as in the flag function but takes env as an additional input and passes the correct substitution for that env in the lambda case. This could even be written as a second mutual recursion for which a second flag function is automatically generated. However, for more complicated cliques of functions, introducing a second similar mutual recursion is rather unwieldy and violates the Don't Repeat Yourself (DRY) principle; the two copies of the mutual recursion must always be maintained in parallel, and it wouldn't be easy in general to remove this duplicated code by generating both versions from one codebase.

An alternative is to use the flag induction of the original mutual recursion but with universal quantification of the variables that need specialized substitutions in some induction hypotheses. That is, instead of proving ( $\mathrm{p} x \mathrm{env}$ ) by induction, we prove (forall env ( $\mathrm{x}$ env)) by induction. This sounds 
Listing 8: Definition and desired theorem about remove-return-last-term

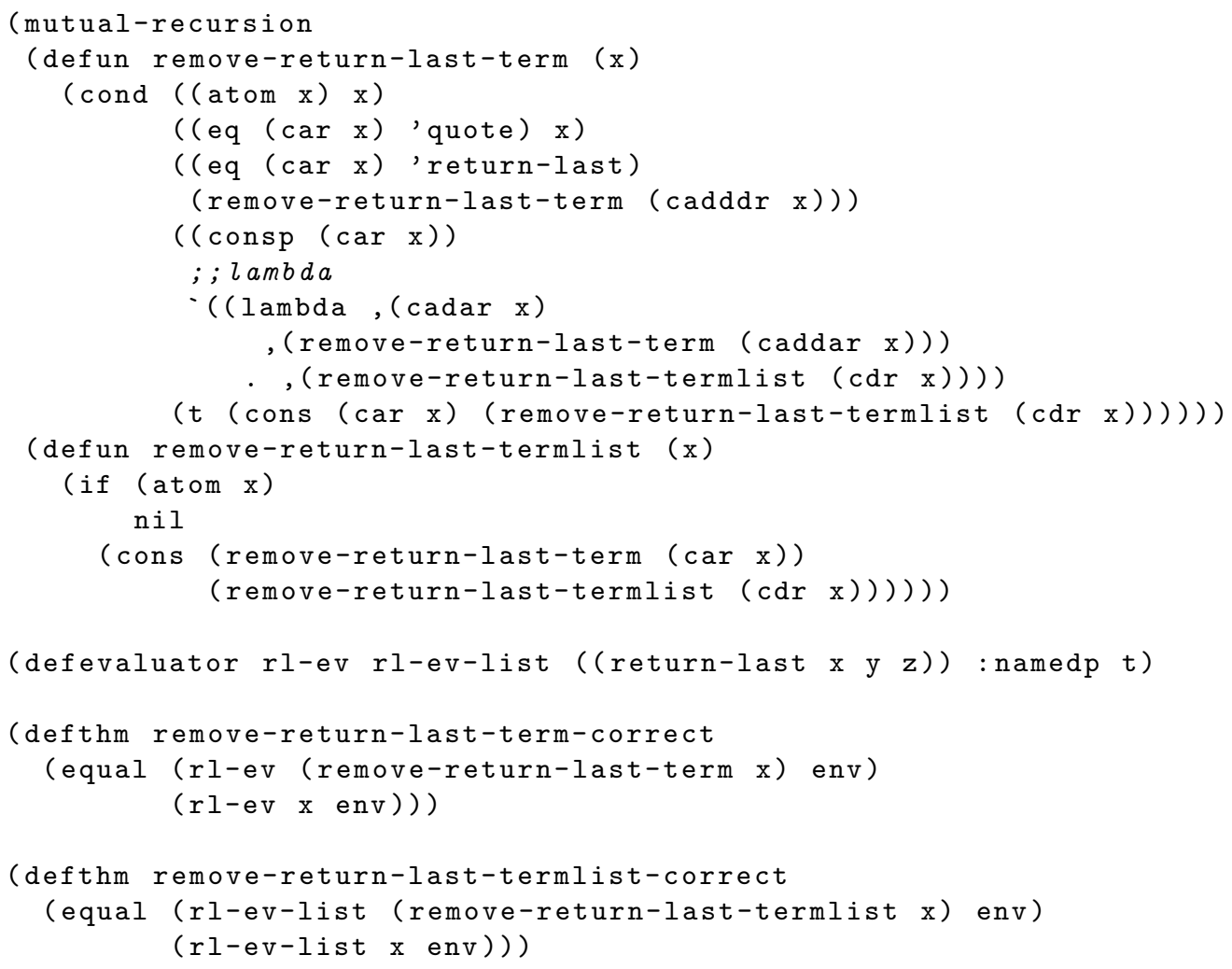


Listing 9: Theorem about remove-return-last-term proved by induction over quantification

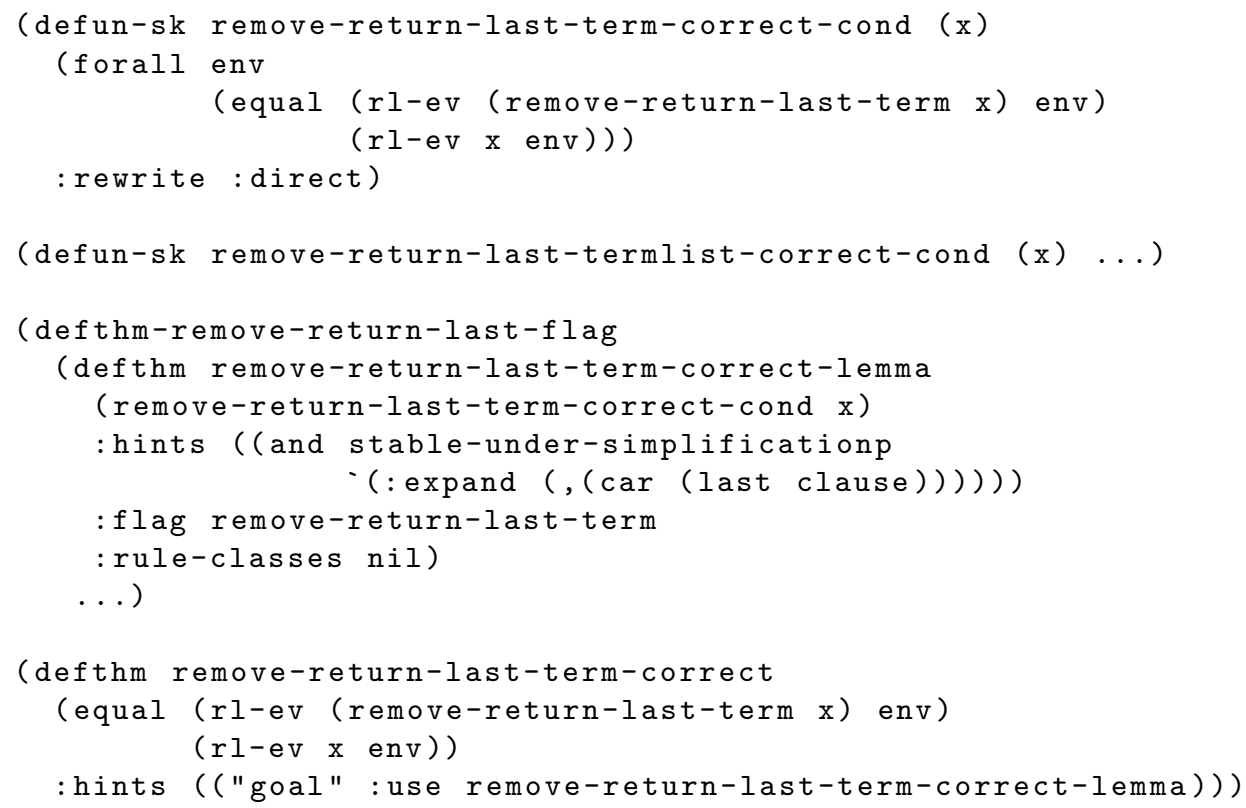

strange in the ACL2 logic where all free variables of a theorem are implicitly universally quantified. However, when we induct on the latter using an induction scheme that applies a substitution to $\mathrm{x}$, we get to assume (forall env ( $\mathrm{p} \sigma(\mathrm{x})$ env)) instead of ( $\mathrm{p} \sigma(\mathrm{x})$ env). To do this we introduce a quantifier function using defun-sk for each of the mutually inductive theorems, prove the quantifier functions true via the flag induction, and then prove the original theorems we wanted as corollaries of those lemmas. We show the process in Listing 9, eliding the termlist versions of the defun-sk, lemma, and final theorem. Of course, there is some lack of DRYness in this method as well, but repeating the theorem bodies is likely preferable to repeating the function definitions, and it would be easier to use macros to streamline this method as well.

Note the stable-under-simplification hint ' (: expand (, (car (last clause))) ) in the inductive lemma. This is often a useful hint for these proofs because it opens the occurrence of the Skolemized function in the conclusion, but not the inductive hypotheses. To prove a universal quantifier introduced with defun-sk true, we want to expand it and prove that its body is true of the witness, whereas if we are assuming it true it is more convenient to leave it unexpanded so that its rewrite rule may be applied.

\section{Proofs using defines and defret-mutual}

The utilities define and def ines add several features to (respectively) defun and mutual-recursion. Their full documentation is in the manual [1], and we will touch on only a few salient features.

The main advantage to define and defines that we exploit to generate mutually inductive theorems is that they store extra data in a table about the functions and mutual recursions they generate. In particular, they allow the return values of functions to be named, and provide a syntax for declaring the types of both return values and formals. This type data is important for defret-mutual-generate, discussed in the next section. But simply naming the return values, especially for functions that return multiple values, allows theorems about such functions to be written much more concisely. The defret 
Listing 10: Defines form for subst-term and evaluation theorem

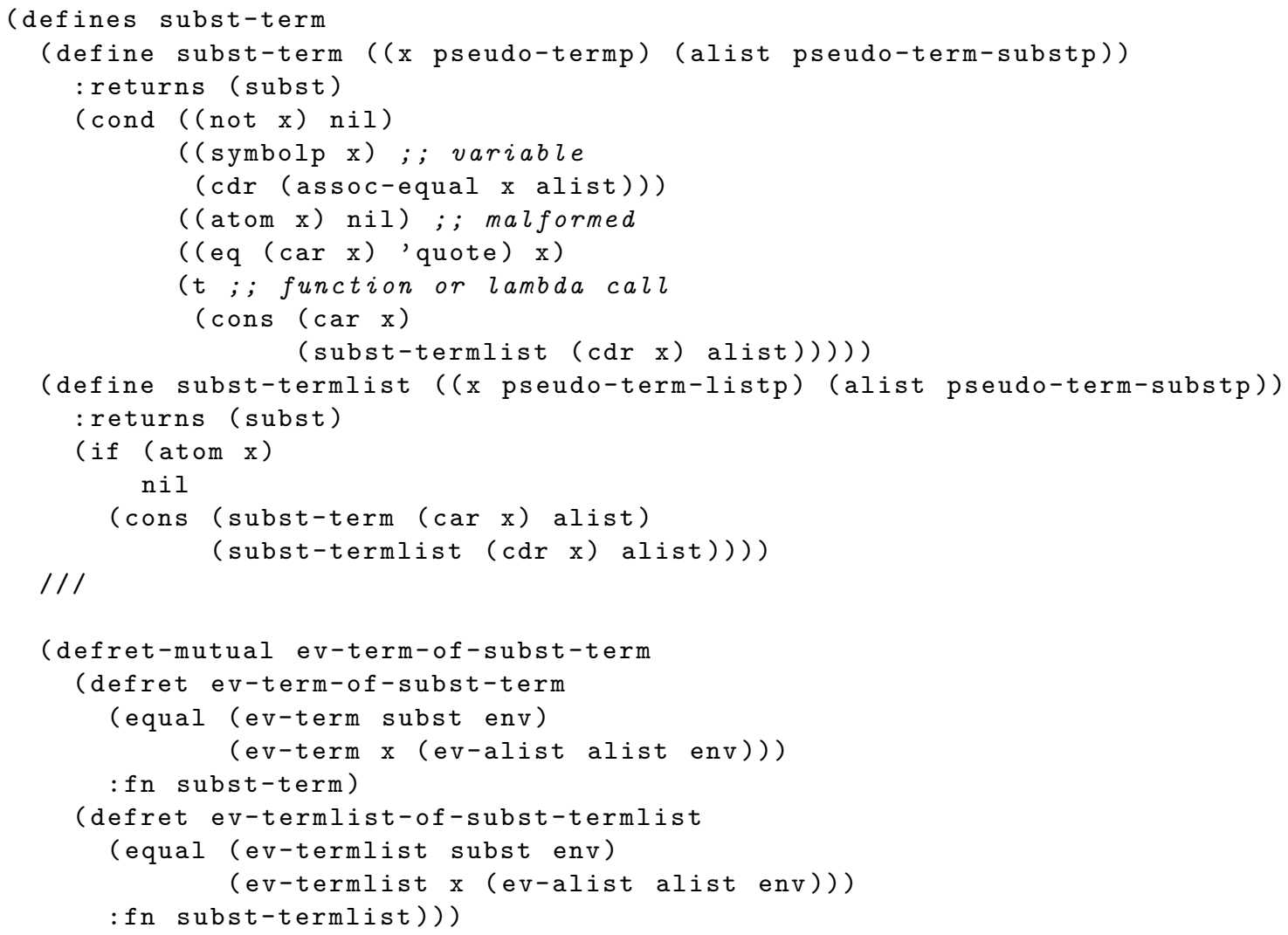

utility produces a defthm form that binds the return values to the call of the function on its formals, for the last function defined with define by default. It also supports various other abbreviations; see the manual [1] for details. For mutual inductions, defret-mutual expands to the flag defthm macro of the mutual recursion most recently introduced with defines, which by default produces an implicit makeflag event.

For our subst-term example, if we recode the function using defines then we can do the same proofs in a defret-mutual form as shown in Listing 10 . Note that because the define forms for each of the functions include a :returns form naming the output of the function subst, the variable subst in the defret forms is implicitly bound to the call of the respective functions.

\section{Automation using defret-mutual-generate}

For proofs about large mutually-recursive cliques, one of the major problems is the usual need to include one theorem per function in order to achieve the correct mutual induction. In proofs about the FGL rewriter [4], the mutually recursive clique in question contains 49 functions, all of which take and return two stobjs, interp-st and state, and most of which have one or two additional arguments and return values. To prove even simple invariants would require writing flag defthm macro forms of well over 300 lines or defret-mutual forms of well over 100 lines.

Previous projects in the ACL2 community books [1] that also ran into this problem have used ad 


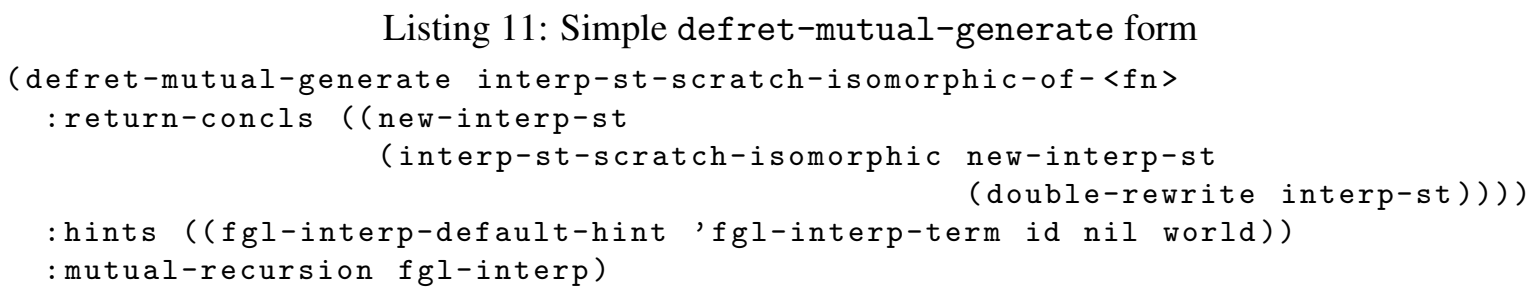

hoc solutions such as custom-built macros to support proofs. For two examples, see the GL interpreter, whose proofs are supported by the macro def-glcp-interp-thm, and the VL expression and statement parsers, which use custom theorem generator functions such as vl-val-when-error-claim, vl-warning-claim, etc. FGL's mutually inductive proofs were instead supported by a more generalpurpose utility, defret-mutual-generate. There are 22 sets of theorems about the FGL rewriter, all generated by this utility. Of those, 17 are mutual inductions and the other five are corollaries of mutually-inductive theorems for which induction isn't needed. The average size of these defretmutual-generate forms is 41 lines. This average is dominated by the final correctness theorem, which is larger because most functions in the mutual recursion need unique correctness statements; this form is 430 lines long, and the average omitting this one is 23 lines.

As a simple example, we show the first defret-mutual-generate form in Listing 11 . The theorem bodies are generated from the :return-concls argument, which essentially says "for each function that has a return value named new-interp-st, prove the following conclusion." In Listing 12 we show two steps of the expansion of the form, heavily elided. First it expands to a defret-mutual form containing 49 defret forms. This then expands, mainly by adding the $b *$ bindings of the return values for each function, to a defthm-fgl-interp-flag containing 49 defthm forms. In both cases we have omitted all but two of the 49 forms from the listing.

A more complicated example is shown in Listing 13 . This form generates a defret-mutual that is 400 lines long, and unlike the previous example the theorems generated aren't all the same. The theorem bodies are generated by applying a set of rules to the function signatures, namely the define formals and returns. These rules are determined by the arguments to the defret-mutual-generate form.

Some of the rules set up by this form could be read in English as follows:

- For each function of the clique that has a formal declared to be type interp-st-bfr-p, add a hypothesis ( $\operatorname{lbfr}-\mathrm{p} x$ ), where $\mathrm{x}$ is the formal name, to the theorem for that function.

- For each function that has a formal named interp-st, add a hypothesis (interp-st-bfrs-ok interp-st).

- For each function that has a return value named $x b f r$, add a conclusion (lbfr-p xbfr newlogicman).

- For each function that has a return value declared to be type fgl-object-p, add a conclusion (lbfr-listp (fgl-object-bfrlist $\mathrm{x}$ ) new-logicman), where $\mathrm{x}$ is the return name.

- To every function's theorem, add the B* bindings for logicman and new-logicman as listed (see the $\mathrm{B} *$ topic in the manual [1]).

- For every function in the list fgl-rewrite-try-rules, etc., add the given scratchobj-case hypothesis. 
Listing 12: Expansions of a defret-mutual-generate form

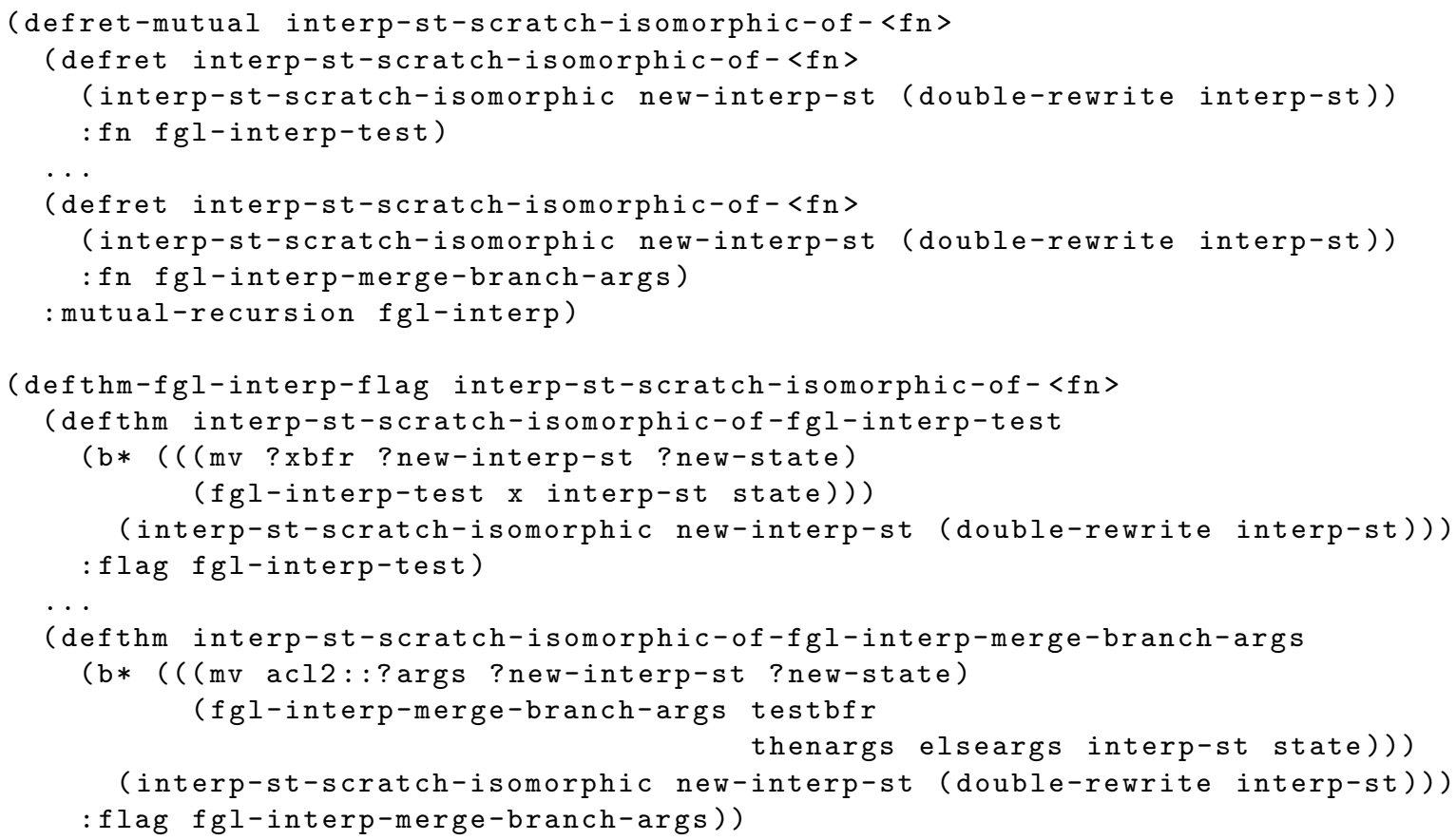

\subsection{Operation of defret-mutual-generate}

Defret-mutual-generate produces a defret-mutual form by applying a set of rules to each function in a mutually recursive clique. These rules may be given directly as arguments to defret-mutualgenerate, but may also be produced by abbreviations such as :formal-hyps and :return-concls, described below.

When applying the rules to each function, each rule has a condition under which it will take effect and a list of actions that update a structure from which a defret form may be generated. This structure contains the following fields:

- Theorem name.

- Top hyps. A list of top-level hypotheses (implicitly conjoined), which apply to the whole conclusion.

- Hyp/conclusion stack. An ordered list containing conclusions (implicitly conjoined) as well as push-hyp and pop-hyp entries; each hypothesis added by a push-hyp entry affects the conclusions listed subsequently until the corresponding occurrence of pop-hyp.

- Bindings. An ordered list of B* bindings to be applied to all hypotheses and conclusions generated.

- Keywords. Keyword/value arguments such as :hints and :rule-classes.

Initially, there are no hypotheses, conclusions, bindings, or keywords in this structure. Rules may add/ push/pop hypotheses and add conclusions, add bindings, change the theorem name, and add keyword arguments. When all the rules have been applied, a defret form is generated from the final structure unless the structure contains no conclusion, in which case it is skipped.

The conditions governing the rules may be a Boolean AND/OR/NOT combination of the following primitive expressions, along with $t$ and $n i l$ : 
Listing 13: Expansions of a defret-mutual-generate form

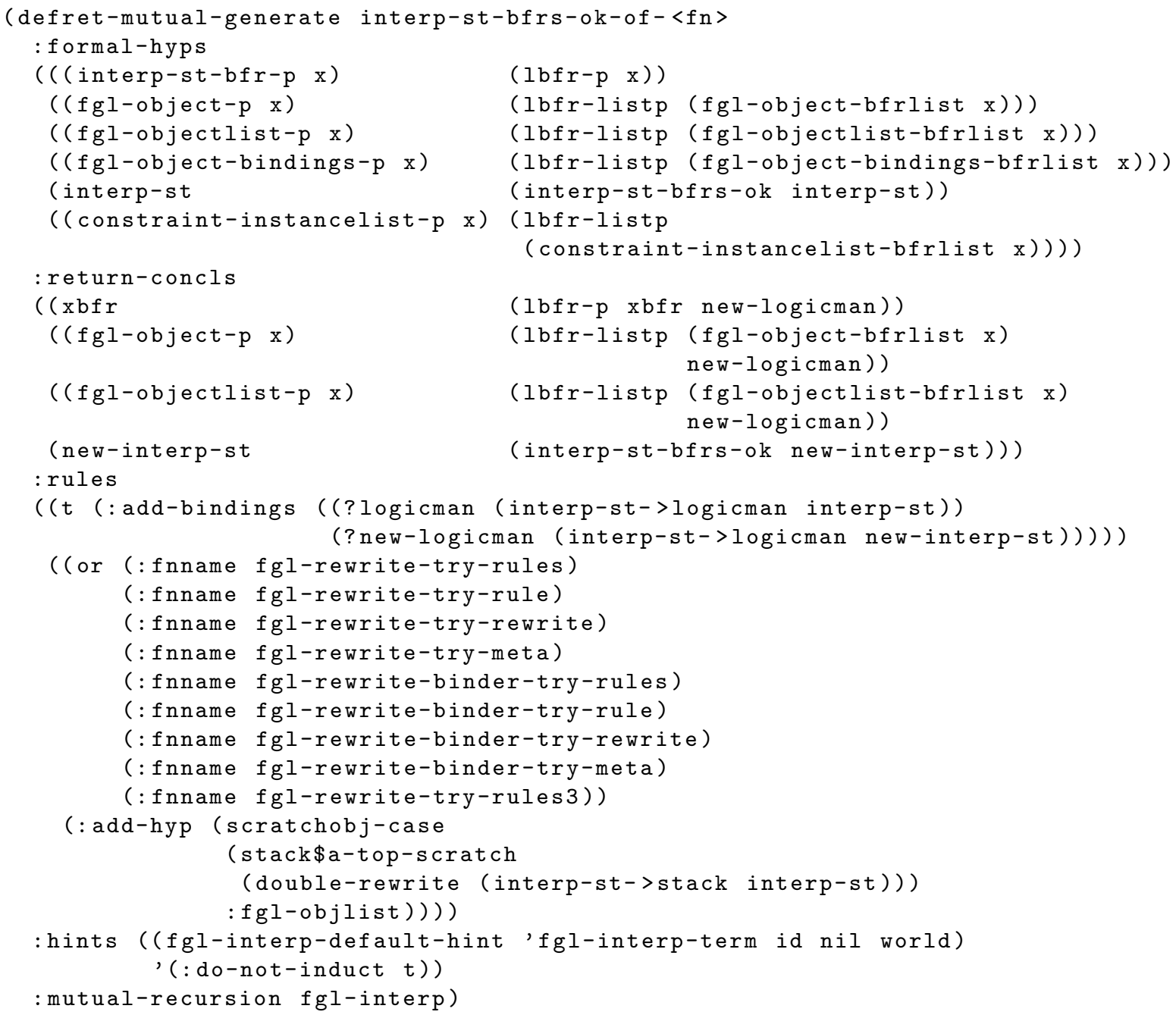


- (: fnname name) checks that the name of the function is name.

- (:has-formal [ :name name ] [ :type type ]) checks that the function has a formal satisfying the listed criteria. The name and type options may be used individually or in combination.

- (:has-return [ : name name ] [ :type type ]) checks that the function has a return value satisfying the listed criteria.

The actions may be any of the following:

- (: add-hyp term) adds term as a top-level hypothesis.

- ( : add-concl term) adds term as a conclusion to the hyp/conclusion stack.

- ( : add-bindings bindings) appends bindings to the end of the current bindings list.

- (: push-hyp term) adds a push-hyp entry term to the hyp/conclusion stack.

- (: pop-hyp) adds a pop-hyp entry to the hyp/conclusion stack, cancelling the effect of the previous push-hyp event on subsequently added conclusions.

- (:each-formal :type type :var var :action action), where action is either an :add-hyp, :push-hyp, :pop-hyp, or : add-concl form, does the given action once for each formal of the given type, substituting the formal name in each case for var in the added hyp/conclusion term.

- (:each-return :type type :var var :action action) is similar to :each-formal but runs instead on each return value.

- (: add-keyword key val) adds the given keyword/value pair to the keywords.

- (:set-thmname template) sets the theorem name to the given template symbol, where any substring $\langle\mathrm{FN}\rangle$ of the template is replaced by the name of the function.

The following keywords generate additional rules from the arguments provided:

- : formal-hyps generates hypotheses based on the names or types of formals. It takes an argument which is a list of elements of the following two forms:

- (name term [ :type type ] ) adds the given term as a top-level hypothesis to the theorem of any function with a formal of the given name. If a type is provided, it will only be added if that formal is of the given type. This translates to a rule with a : has-formal condition and an : add-hyp action.

- ((type name) term) adds the given term as a top-level hypothesis for every formal of the given type, binding that formal to name. This translates to an : each-formal : add-hyp rule under condition $t$.

- :return-concls is analogous to : formal-hyps, generating conclusions based on the names or types of return values. The same forms of argument are accepted.

- : function-keys adds keywords to the theorems corresponding to function names. It accepts an argument which is a list of entries (fnname key val ...). 


\section{Conclusion}

The utilities described in this paper are effective in reducing the boilerplate and code duplication that is otherwise necessary for proving mutually inductive theorems. In developing the FGL rewriter, which is a 49-function mutually recursive clique, these tools were used to great effect in proving the necessary theorems, including its semantic correctness with respect to an evaluator. We have made many revisions to the FGL rewriter since its correctness proofs were first completed, including adding and removing several functions from the mutual recursion. However, because we use defret-mutual-generate to produce the theorems about this mutual recursion, we usually find that the only one that needs to be significantly updated is the final semantic correctness theorem. We have also found it to be advantageous to split functions from the FGL rewriter into smaller mutually-recursive parts, since this makes each step of the inductive proof smaller. Normally, this would mean that proof scripts would need to grow larger in order to accommodate the new functions of the clique, but again we find that most of the defret-mutual-generate forms that generate our proofs need no modification.

\section{References}

[1] ACL2 Community (Accessed: 2020): ACL2+Books Documentation. Available at http://www . cs . utexas . edu/users/moore/acl2/manuals/current/manual/index.html.

[2] Robert S. Boyer \& J Strother Moore (1984): A mechanical proof of the unsolvability of the halting problem. Journal of the ACM (JACM) 31(3), pp. 441-458, doi:10.1145/828.1882.

[3] Warren A. Hunt Jr., Matt Kaufmann, Robert Bellarmine Krug, J. Strother Moore \& Eric Whitman Smith (2005): Meta Reasoning in ACL2. In Joe Hurd \& Tom Melham, editors: Theorem Proving in Higher Order Logics, Springer Berlin Heidelberg, Berlin, Heidelberg, pp. 163-178, doi: 10.1007/11541868_11.

[4] Sol Swords (Accessed: 2020): FGL source distribution. Available at https://github.com/ac12/ac12/ tree/master/books/centaur/fgl. 